\title{
Generalized $\mathrm{C}_{\lambda}$-Rate Sequence Spaces of Difference Sequence Defined by a Modulus Function in a Locally Convex Space
}

\author{
B.Özaltın, İ. Dağadur* \\ Department of Mathematics, Faculty of Science and Literature, Mersin University, 33343, Mersin, Turkey
}

Copyright (C)2015 Horizon Research Publishing All rights reserved.

\begin{abstract}
The idea of difference sequence spaces was introduced by Kizmaz [14] and this concept was generalized by Et and Çolak [6] . Recently the difference sequence spaces have been studied in (see, [3], [7], [17], [18]). The purpose of this article is to introduce the sequence spaces $C_{c_{0 \pi}}^{\lambda}\left(\Delta^{m}, f, p, q\right), C_{c_{\pi}}^{\lambda}\left(\Delta^{m}, f, p, q\right)$ and $C_{\left(\underline{\ell}_{\infty}\right)}^{\lambda}\left(\Delta^{m}, f, p, q\right)$ using a modulus function $f$ and more general $C_{\lambda}$ - method in viev of Armitage and Maddox [2]. Several properties of these spaces, and some inclusion relations have been examined.
\end{abstract}

Keywords FK-spaces, Modulus Function, Rate Sequence Space, Difference Sequence, $C_{\lambda}$-Summability Method

2000 Mathematics Subject Classification: 40C05, 40D25, 40G05, 42A05, 42A10

\section{Introduction}

The notion of a modulus was introduced by Nakano [19]. We recall that a modulus $f$ is a function from $[0, \infty)$ to $[0, \infty)$ such that

i) $f(x)=0$ if and only if $x=0$,

ii) $f(x+y) \leq f(x)+f(y)$ for $x, y \geq 0$,

iii) $f$ is increasing,

iv) $f$ is continuous from the right at 0 .

It follows that $f$ must be continuous everywhere on $[0, \infty)$. Maddox [15] and Ruckle [22] used a modulus function to construct some sequence spaces. Later on using a modulus different sequence spaces have been studied by Altın and Et [1], Et [5], Nuray and Savas [20], Tripathy and Chandra [26] and many others.

The notion of difference sequence spaces was introduced by Kızmaz [14] and the notion was generalized by Et and Çolak [6]. Recently the difference sequence spaces have been studied in $([3],[7],[10],[17],[18])$.

Let $w$ be the set of all sequences of real or complex numbers and $\ell_{\infty}, c$ and $c_{0}$ be respectively the Banach spaces of bounded, convergent and null sequences $x=\left(x_{k}\right)$ with the usual norm $\|x\|_{\infty}=\sup \left|x_{k}\right|$, where $k \in \mathbb{N}=\{1,2, \ldots\}$, the set of positive integers. Also by $b s, c s, \ell_{1}$ and $\ell_{p}$; we denote the spaces of all bounded, convergent, absolutely and $p$-absolutely convergent series, respectively.

A sequence space $E$ with a linear topology is called a $K$-space provided each of the maps $p_{i}: E \rightarrow \mathbb{C}$ defined by $p_{i}(x)=x_{i}$ is continuous for each $i \in \mathbb{N}$, where $\mathbb{C}$ denotes the complex field. A $K$-space $E$ is called an $F K-$ space provided $E$ is a complete linear metric space. An $F K$-space whose topology is normable is called a $B K-$ space. The basic properties of $F K$-spaces may be found in ([27], [28], [29], [30]).

Let $\pi=\left(\pi_{n}\right)$ be a sequence of positive numbers i.e, $\pi_{n}>0, \forall n \in \mathbb{N}$ and $X$ is an $F K-$ space. We shall consider the sets of sequences $x=\left(x_{n}\right)$

$$
X_{\pi}=\left\{x \in w:\left(\frac{x_{n}}{\pi_{n}}\right) \in X\right\} .
$$

The set $X_{\pi}$ may be considered as $F K$-space. We shall call them as rate spaces (see, [12] and [13]). 
Let $F$ be an infinite subset of $\mathbb{N}$ and $F$ as the range of a strictly increasing sequence of positive integers, say $F=$ $\{\lambda(n)\}_{n=1}^{\infty}$. The Cesáro submethod $C_{\lambda}$ is defined as

$$
\left(C_{\lambda} x\right)_{n}=\frac{1}{\lambda(n)} \sum_{k=1}^{\lambda(n)} x_{k},(n=1,2, \ldots),
$$

where $\left\{x_{k}\right\}$ is a sequence of a real or complex numbers. Therefore, the $C_{\lambda}$-method yields a subsequence of the Cesáro method $C_{1}$, and hence it is regular for any $\lambda . C_{\lambda}$ is obtained by deleting a set of rows from Cesáro matrix. If $\lambda(n)=n$ is taken, then $C_{\lambda}=C_{1}$ is obteined. On a range of sequences

$$
\lim _{n}\left(C_{\lambda} x\right)_{n}:=\lim _{n}\left(C_{1} x\right)_{n},
$$

we will write $C_{\lambda} \sim C_{1}$. The basic properties of $C_{\lambda}$-method can be found in [2] and [21].

We need the following inequality throughout the paper. Let $p=\left(p_{k}\right)$ be a sequence of positive real numbers with $G=\sup _{k} p_{k}$ and $D=\max \left(1,2^{G-1}\right)$. Then, it is well known that for all $a_{k}, b_{k} \in \mathbb{C}$, the field of complex numbers, for all $k \in \mathbb{N}$,

$$
\left|a_{k}+b_{k}\right|^{p_{k}} \leq D\left(\left|a_{k}\right|^{p_{k}}+\left|b_{k}\right|^{p_{k}}\right) .
$$

Also for any complex $\mu$,

$$
\mu^{p_{k}} \leq \max \left(1, \mu^{G}\right)
$$

see in $[16]$.

Let $X$ be a sequence space. Then $X$ is called;

i) Solid (or normal) if $\left(\alpha_{k} x_{k}\right) \in X$ whenever $\left(x_{k}\right) \in X$ for all sequences $\left(\alpha_{k}\right)$ of scalars with $\left|\alpha_{k}\right| \leq 1$,

ii) Symmetric if $\left(x_{k}\right) \in X$ implies $\left(x_{\pi(k)}\right) \in X$, where $\pi$ is a permutation of $\mathbb{N}$,

iii) Sequence algebra if $X$ is closed under multiplication.

\section{Main Results}

In this section we give the main results of this paper.

Definition 2.1 Let $f$ be a modulus function, $X$ be a locally convex Hausdorff topological linear space whose topology is determined by a set $Q$ of continuous seminorms $q$ and $p=\left(p_{k}\right)$ be a sequence of positive real numbers. The symbol $w(X)$ denotes the space of all sequences defined over $X$.

$$
\left.\begin{array}{rl}
C_{c_{0}}^{\lambda}\left(\Delta^{m}, f, p, q\right) & =\left\{\begin{array}{c}
x \in w(X): \frac{1}{\lambda(n)} \sum_{k=1}^{\lambda(n)}\left[f\left(q\left(\Delta^{m} \frac{x_{k}}{\pi_{k}}\right)\right)\right]^{p_{k}} \longrightarrow 0 \\
\text { as } n \longrightarrow \infty
\end{array}\right\} \\
C_{c_{\pi}}^{\lambda}\left(\Delta^{m}, f, p, q\right) & =\left\{\begin{array}{c}
x \in w(X): \frac{1}{\lambda(n)} \sum_{k=1}^{\lambda(n)}\left[f\left(q\left(\Delta^{m} \frac{x_{k}}{\pi_{k}}-L\right)\right)\right]^{p_{k}} \longrightarrow 0 \\
\text { as } n \longrightarrow \infty, \text { for some } L
\end{array}\right\} \\
C_{\left(\ell_{\infty}\right)_{\pi}}^{\lambda}\left(\Delta^{m}, f, p, q\right) & =\left\{x \in w(X): \sup _{n} \frac{1}{\lambda(n)} \sum_{k=1}^{\lambda(n)}\left[f\left(q\left(\Delta^{m} \frac{x_{k}}{\pi_{k}}\right)\right)\right]^{p_{k}}<\infty\right.
\end{array}\right\},
$$

where $\Delta^{0} x=\frac{x_{k}}{\pi_{k}}, \Delta^{m} x=\left(\Delta^{m-1} \frac{x_{k}}{\pi_{k}}-\Delta^{m-1} \frac{x_{k+1}}{\pi_{k+1}}\right)$ and $\Delta^{m} x_{k}=\sum_{v=0}^{m}(-1)^{v}\left(\begin{array}{c}m \\ v\end{array}\right) \frac{x_{k+v}}{\pi_{k+v}}$. For $f(x)=x$ we shall write $C_{c_{0 \pi}}^{\lambda}\left(\Delta^{m}, p, q\right), C_{c_{\pi}}^{\lambda}\left(\Delta^{m}, p, q\right)$ and $C_{\left(\ell_{\infty}\right)_{\pi}}^{\lambda}\left(\Delta^{m} p, q\right)$ instead of $C_{c_{0 \pi}}^{\lambda}\left(\Delta^{m}, f, p, q\right), C_{c_{\pi}}^{\lambda}\left(\Delta^{m}, f, p, q\right)$ and $C_{\left(\ell_{\infty}\right)_{\pi}}^{\lambda}\left(\Delta^{m}, f, p, q\right)$ respecxtively.

The proof of each of the following results is fairly straightforward, so we choose to state these results without proof.

Theorem 2.1. Let $p=\left(p_{k}\right)$ be bounded, then $C_{c_{\pi}}^{\lambda}\left(\Delta^{m}, f, p, q\right), C_{c_{0 \pi}}^{\lambda}\left(\Delta^{m}, f, p, q\right)$ and $C_{\left(\ell_{\infty}\right)_{\pi}}^{\lambda}\left(\Delta^{m}, f, p, q\right)$ are linear spaces.

Theorem 2.2. $C_{c_{0 \pi}}^{\lambda}\left(\Delta^{m}, f, p, q\right)$ is a paranormed space (not totally paranormed), paranormed by

$$
g_{\Delta}(x)=\sup _{n}\left\{\frac{1}{\lambda(n)} \sum_{k=1}^{\lambda(n)}\left[f\left(q\left(\Delta^{m} \frac{x_{k}}{\pi_{k}}\right)\right)\right]^{p_{k}}\right\}^{\frac{1}{M}}
$$

where $G=\sup _{k} p_{k}<\infty$ ve $M=\max (1, G)$. 
Theorem 2.3. Let $f, f_{1}, f_{2}$ are modulus functions and

$$
0<h=\inf p_{k} \leq p_{k} \leq \sup _{k} p_{k}=G<\infty
$$

then

(i) $C_{c_{0 \pi}}^{\lambda}\left(\Delta^{m}, f_{1}, p, q\right) \subseteq C_{c_{0 \pi}}^{\lambda}\left(\Delta^{m}, f \circ f_{1}, p, q\right)$,

(ii) $C_{c_{0 \pi}}^{\lambda^{\pi}}\left(\Delta^{m}, f_{1}, p, q\right) \cap C_{c_{0 \pi}}^{\lambda}\left(\Delta^{m}, f_{2}, p, q\right) \subseteq C_{c_{0 \pi}}^{\lambda}\left(\Delta^{m}, f_{1}+f_{2}, p, q\right)$.

Proof . (i) Let $x=\left(\frac{x_{k}}{\pi_{k}}\right) \in C_{c_{0 \pi}}^{\lambda}\left(\Delta^{m}, f_{1}, p, q\right)$. Let $\varepsilon>0$ and choose $\delta$ with $0<\delta<1$ such that $f(t)<\varepsilon$ for $0 \leq t \leq \delta$. Write $y_{k}=f_{1}\left(q\left(\Delta^{m} \frac{x_{k}}{\pi_{k}}\right)\right)$ and consider

$$
\sum_{k=1}^{\lambda(n)}\left[f\left(y_{k}\right)\right]^{p_{k}}=\sum_{1}\left[f\left(y_{k}\right)\right]^{p_{k}}+\sum_{2}\left[f\left(y_{k}\right)\right]^{p_{k}}
$$

where the first summation is over $y_{k} \leq \delta$ and the second over $y_{k}>\delta$. Since $f$ is continuous, we get

$$
\sum_{1}\left[f\left(y_{k}\right)\right]^{p_{k}}<\lambda(n) \max \left(\varepsilon^{h}, \varepsilon^{G}\right)
$$

and for $y_{k}>\delta$ we use the fact that

$$
y_{k}<\frac{y_{k}}{\delta}<1+\frac{y_{k}}{\delta}
$$

By the definition of $f$ we have for $y_{k}>\delta$,

$$
f\left(y_{k}\right) \leq f(1)\left[1+\left(\frac{y_{k}}{\delta}\right)\right] \leq 2 f(1) \frac{y_{k}}{\delta} .
$$

Hence

$$
\frac{1}{\lambda(n)} \sum_{2}\left[f\left(y_{k}\right)\right]^{p_{k}} \leq \max \left(1,\left(\frac{2 f(1)}{\delta}\right)^{G}\right) \frac{1}{\lambda(n)} \sum_{k=1}^{\lambda(n)}\left[y_{k}\right]^{p_{k}} \longrightarrow 0 .
$$

By (3) and (4) we have $C_{c_{0 \pi}}^{\lambda}\left(\Delta^{m}, f_{1}, p, q\right) \subseteq C_{c_{0 \pi}}^{\lambda}\left(\Delta^{m}, f \circ f_{1}, p, q\right)$.

(ii) Let $\left(\frac{x_{k}}{\pi_{k}}\right) \in C_{c_{0 \pi}}^{\lambda}\left(\Delta^{m}, f_{1}, p, q\right) \cap C_{c_{0 \pi}}^{\lambda}\left(\Delta^{m}, f_{2}, p, q\right)$. Then there exist $f_{1}$ and $f_{2}$ such that

$$
\begin{aligned}
& \frac{1}{\lambda(n)} \sum_{k=1}^{\lambda(n)}\left[f_{1}\left(q\left(\Delta^{m} \frac{x_{k}}{\pi_{k}}\right)\right)\right]^{p_{k}} \longrightarrow 0 \text { as } n \longrightarrow \infty, \\
& \frac{1}{\lambda(n)} \sum_{k=1}^{\lambda(n)}\left[f_{2}\left(q\left(\Delta^{m} \frac{x_{k}}{\pi_{k}}\right)\right)\right]^{p_{k}} \longrightarrow 0 \text { as } n \longrightarrow \infty .
\end{aligned}
$$

Then using (1) it can be shown that

$$
\begin{aligned}
& \frac{1}{\lambda(n)} \sum_{k=1}^{\lambda(n)}\left[\left(f_{1}+f_{2}\right)\left(q\left(\Delta^{m} \frac{x_{k}}{\pi_{k}}\right)\right)\right]^{p_{k}} \\
= & \frac{1}{\lambda(n)} \sum_{k=1}^{\lambda(n)}\left[f_{1}\left(q\left(\Delta^{m} \frac{x_{k}}{\pi_{k}}\right)\right)+f_{2}\left(q\left(\Delta^{m} \frac{x_{k}}{\pi_{k}}\right)\right)\right]^{p_{k}} \\
\leq & D \frac{1}{\lambda(n)} \sum_{k=1}^{\lambda(n)}\left[f_{1}\left(q\left(\Delta^{m} \frac{x_{k}}{\pi_{k}}\right)\right)\right]^{p_{k}}+D \frac{1}{\lambda(n)} \sum_{k=1}^{\lambda(n)}\left[f_{2}\left(q\left(\Delta^{m} \frac{x_{k}}{\pi_{k}}\right)\right)\right]^{p_{k}} \\
\longrightarrow & 0 \text { as } n \longrightarrow \infty
\end{aligned}
$$

By (5) and (6), then $\frac{1}{\lambda(n)} \sum_{k=1}^{\lambda(n)}\left[\left(f_{1}+f_{2}\right)\left(q\left(\Delta^{m} \frac{x_{k}}{\pi_{k}}\right)\right)\right]^{p_{k}} \longrightarrow 0$ as $n \longrightarrow \infty$. Therefore $\left(\frac{x_{k}}{\pi_{k}}\right) \in C_{c_{0 \pi}}^{\lambda}\left(\Delta^{m}, f_{1}+f_{2}, p, q\right)$. Hence

$$
C_{c_{0 \pi}}^{\lambda}\left(\Delta^{m}, f_{1}, p, q\right) \cap C_{c_{0 \pi}}^{\lambda}\left(\Delta^{m}, f_{2}, p, q\right) \subseteq C_{c_{0 \pi}}^{\lambda}\left(\Delta^{m}, f_{1}+f_{2}, p, q\right) .
$$

The proof of the following result is a routune work in view of the above Theorem.

Corallary 2.4. $f, f_{1}, f_{2}$ are modulus functions. Then

(i) $C_{c_{\pi}}^{\lambda}\left(\Delta^{m}, f_{1}, p, q\right) \subseteq C_{c_{\pi}}^{\lambda}\left(\Delta^{m}, f \circ f_{1}, p, q\right)$,

(ii) $C_{c_{\pi}}^{\lambda}\left(\Delta^{m}, f_{1}, p, q\right) \cap C_{c_{\pi}}^{\lambda}\left(\Delta^{m}, f_{2}, p, q\right) \subseteq C_{c_{\pi}}^{\lambda}\left(\Delta^{m}, f_{1}+f_{2}, p, q\right)$ 
(iii) $C_{\left(\ell_{\infty}\right)_{\pi}}^{\lambda}\left(\Delta^{m}, f_{1}, p, q\right) \subseteq C_{\left(\ell_{\infty}\right)_{\pi}}^{\lambda}\left(\Delta^{m}, f \circ f_{1}, p, q\right)$

(iv) $C_{\left(\ell_{\infty}\right)_{\pi}}^{\lambda}\left(\Delta^{m}, f_{1}, p, q\right) \cap C_{\left(\ell_{\infty}\right)_{\pi}}^{\lambda}\left(\Delta^{m}, f_{2}, p, q\right) \subseteq C_{\left(\ell_{\infty}\right)_{\pi}}^{\lambda}\left(\Delta^{m}, f_{1}+f_{2}, p, q\right)$

The following result is a routune work.

Proposition 2.5. $C_{c_{\pi}}^{\lambda}\left(\Delta^{m-1}, f, p, q\right) \subseteq C_{c_{\pi}}^{\lambda}\left(\Delta^{m}, f, p, q\right)$.

Theorem 2.6. Let $m \geq 1$, then the following inclusions are strict.

(i) $C_{c_{0 \pi}}^{\lambda}\left(\Delta^{m-1}, f, q\right) \subseteq C_{c_{0 \pi}}^{\lambda}\left(\Delta^{m}, f, q\right)$

(ii) $C_{c_{\pi}}^{\lambda}\left(\Delta^{m-1}, f, q\right) \subseteq C_{c_{\pi}}^{\lambda}\left(\Delta^{m}, f, q\right)$

(iii) $C_{\left(\ell_{\infty}\right)_{\pi}}^{\lambda}\left(\Delta^{m-1}, f, q\right) \subseteq C_{\left(\ell_{\infty}\right)_{\pi}}^{\lambda}\left(\Delta^{m}, f, q\right)$

Proof. We prove the case $(i)$ only. The other cases follow in a similar way. Let $\left(\frac{x_{k}}{\pi_{k}}\right) \in C_{c_{0 \pi}}^{\lambda}\left(\Delta^{m-1}, f, q\right)$. Then we have

$$
\frac{1}{\lambda(n)} \sum_{k=1}^{\lambda(n)} f\left(q\left(\Delta^{m-1} \frac{x_{k}}{\pi_{k}}\right)\right) \longrightarrow 0 \text { as } n \longrightarrow \infty .
$$

By the definition of $f$ and $q$, we have

$$
\begin{aligned}
& \frac{1}{\lambda(n)} \sum_{k=1}^{\lambda(n)} f\left(q\left(\Delta^{m} \frac{x_{k}}{\pi_{k}}\right)\right) \\
\leq & \frac{1}{\lambda(n)} \sum_{k=1}^{\lambda(n)} f\left(q\left(\Delta^{m-1} \frac{x_{k}}{\pi_{k}}-\Delta^{m-1} \frac{x_{k+1}}{\pi_{k+1}}\right)\right) \\
\leq & \frac{1}{\lambda(n)} \sum_{k=1}^{\lambda(n)} f\left(q\left(\Delta^{m-1} \frac{x_{k}}{\pi_{k}}\right)\right)+\frac{1}{\lambda(n)} \sum_{k=1}^{\lambda(n)} f\left(q\left(\Delta^{m-1} \frac{x_{k+1}}{\pi_{k+1}}\right)\right) \\
\longrightarrow & 0 \text { as } n \longrightarrow \infty .
\end{aligned}
$$

This completes the proof. In general $C_{c_{\pi}}^{\lambda}\left(\Delta^{i}, f, q\right) \subseteq C_{c_{\pi}}^{\lambda}\left(\Delta^{m}, f, q\right)$ for all $i=1,2,3, \ldots, m-1$ and the inclusion is strict. To show that the inclusion is strict, consider the following example.

Example 2.7. Let $X=\mathbb{C}, f(x)=x$, and $q(x)=|x|$. Consider the sequences $\left(x_{k}\right)=\left(k^{m+\alpha}\right)$ and $\left(\pi_{k}\right)=\left(k^{\alpha+1}\right)$, where $x=\left(\frac{x_{k}}{\pi_{k}}\right)$ and $m \in \mathbb{N}, \alpha \in \mathbb{R}$. Then $x \in C_{c_{0 \pi}}^{\lambda}\left(\Delta^{m}, f, q\right)$ but $x \notin C_{c_{0 \pi}}^{\lambda}\left(\Delta^{m-1}, f, q\right)$, since $\Delta^{m} \frac{x_{k}}{\pi_{k}}=0, \Delta^{m-1} \frac{x_{k}}{\pi_{k}}=$ $(-1)^{m-1}(m-1)$ ! for $\forall k \in \mathbb{N}$.

Theorem 2.8. For any two sequence $p=\left(p_{k}\right)$ and $t=\left(t_{k}\right)$ of positive real numbers and any two seminorms $q_{1}, q_{2}$ we have

(i) $C_{c_{0 \pi}}^{\lambda}\left(\Delta^{m}, f, p, q_{1}\right) \cap C_{c_{0 \pi}}^{\lambda}\left(\Delta^{m}, f, t, q_{2}\right) \neq \emptyset$

(ii) $C_{c_{\pi}}^{\lambda^{\pi}}\left(\Delta^{m}, f, p, q_{1}\right) \cap C_{c_{\pi}}^{\lambda}\left(\Delta^{m}, f, t, q_{2}\right) \neq \emptyset$

(iii) $C_{\left(\ell_{\infty}\right)_{\pi}}^{\lambda}\left(\Delta^{m}, f, p, q_{1}\right) \cap C_{\left(\ell_{\infty}\right)_{\pi}}^{\lambda}\left(\Delta^{m}, f, t, q_{2}\right) \neq \emptyset$.

Proof. Since the zero element belongs to each of the above classes of sequences, thus the intersection is non empty.

The following result is a consequence of Theorem 2. $3(i)$ and Corollary 2. $4(i)$ and $(i i i)$.

Proposition 2.9. Let $f$ be a modulus function. Then

(i) $C_{c_{0 \pi}}^{\lambda}\left(\Delta^{m}, p, q\right) \subseteq C_{c_{0 \pi}}^{\lambda}\left(\Delta^{m}, f, p, q\right)$,

(ii) $C_{c_{\pi}}^{\lambda^{\pi}}\left(\Delta^{m}, p, q\right) \subseteq C_{c_{\pi}}^{\lambda^{\pi}}\left(\Delta^{m}, f, p, q\right)$,

(iii) $C_{\left(\ell_{\infty}\right)_{\pi}}^{\lambda}\left(\Delta^{m}, p, q\right) \subseteq C_{\left(\ell_{\infty}\right)_{\pi}}^{\lambda}\left(\Delta^{m}, f, p, q\right)$.

Theorem 2.10. Let $0<p_{k} \leq r_{k}$ and $\left(\frac{r_{k}}{p_{k}}\right)$ be bounded, then

$$
C_{c_{\pi}}^{\lambda}\left(\Delta^{m}, f, r, q\right) \subseteq C_{c_{\pi}}^{\lambda}\left(\Delta^{m}, f, p, q\right) .
$$

Proof. Omitted.

Theorem.2.11. The sequence spaces $C_{c_{0 \pi}}^{\lambda}\left(\Delta^{m}, f, p, q\right), C_{c_{\pi}}^{\lambda}\left(\Delta^{m}, f, p, q\right)$ and $C_{\left(\ell_{\infty}\right)_{\pi}}^{\lambda}\left(\Delta^{m}, f, p, q\right)$ are neither solid nor symmetric, nor sequence algebras for $m \geq 1$.

Proof. Let $m=1, p_{k}=1$ for all $k \in \mathbb{N}, f(x)=x$ and $q(x)=|x|$. If the sequences $\left(x_{k}\right)=\left(k^{n+1}\right)$ and $\left(\pi_{k}\right)=\left(k^{n}\right)$ are taken, then the sequence $\left(\frac{x_{k}}{\pi_{k}}\right)$ belongs to $C_{\left(\ell_{\infty}\right)_{\pi}}^{\lambda}(\Delta)$ and $C_{c_{\pi}}^{\lambda}(\Delta)$, where $n \in \mathbb{R}$. Let $\alpha_{k}=(-1)^{k}$, then $\left(\alpha_{k} x\right)$ does not belong to $C_{\left(\ell_{\infty}\right)_{\pi}}^{\lambda}(\Delta)$ and $C_{c_{\pi}}^{\lambda}(\Delta)$. Hence $C_{c_{\pi}}^{\lambda}\left(\Delta^{m}, f, p, q\right)$ and $C_{\left(\ell_{\infty}\right)_{\pi}}^{\lambda}\left(\Delta^{m}, f, p, q\right)$ are not solid. The other cases can be proved on considering similar examples. 


\section{Results Related to Statical Convergence}

Fast [8] and (independently) Schoenberg [24] introduced the notion of statistical convergence. The idea depends on the density of subsets of the set $\mathbb{N}$ of natural numbers. The density of $E$ a subset of $\mathbb{N}$ is defined by $\delta(E)=\lim _{n \rightarrow \infty} \frac{1}{n} \sum_{k=1}^{n} \chi_{E}(k)$ provided the limit exists, where $\chi_{E}$ is the characteristic function of $E$. A sequence $x=\left(x_{k}\right)$ is called statistically convergent to a number $L$, if for every $\varepsilon>0, \delta\left\{k \in \mathbb{N}:\left|x_{k}-L\right| \geq \varepsilon\right\}=0$. Later on it was further investigated from the sequence space point of view and linked with summability theory by Fridy [9] and Salat [23] and many others.

Definition 3.1. Let $\pi=\left(\pi_{k}\right)$ be a sequence of pozitif numbers. A sequence $x=\left(x_{k}\right)$ is said to be $\Delta_{q}^{m}$-statistically convergent to $L \in X$ if for all $q \in Q$ and any $\varepsilon>0$,

$$
\lim _{n \rightarrow \infty} \frac{1}{\lambda(n)}\left|\left\{k \leq \lambda(n): q\left(\Delta^{m} \frac{x_{k}}{\pi_{k}}-L\right) \geq \varepsilon\right\}\right|=0,
$$

where the vertical bars indicate the number of elements in the closed set. In this case we write $\left(\frac{x_{k}}{\pi_{k}}\right) \longrightarrow L\left(S\left(\Delta_{q}^{m}\right)\right)$.

Theorem 3.2. Let $f$ be a modulus function and $\sup _{k} p_{k}=G<\infty$. Then $C_{c_{\pi}}^{\lambda}\left(\Delta^{m}, f, p, q\right) \subset S\left(\Delta_{q}^{m}\right)$.

Proof. Let $x \in C_{c_{\pi}}^{\lambda}\left(\Delta^{m}, f, p, q\right)$. Take $q \in Q, \varepsilon>0$ and let $\sum_{1}$ denote the sum over $k \leq \lambda(n)$ with $q\left(\Delta^{m} \frac{x_{k}}{\pi_{k}}-L\right) \geqslant \varepsilon$ and $\sum_{2}$ denote the sum over $k \leq \lambda(n)$ with $q\left(\Delta^{m} \frac{x_{k}}{\pi_{k}}-L\right)<\varepsilon$. Then

$$
\begin{aligned}
& \frac{1}{\lambda(n)} \sum_{k=1}^{\lambda(n)}\left[f\left(q\left(\Delta^{m} \frac{x_{k}}{\pi_{k}}-L\right)\right)\right]^{p_{k}} \\
= & \frac{1}{\lambda(n)}\left(\sum_{1}\left[f\left(q\left(\Delta^{m} \frac{x_{k}}{\pi_{k}}-L\right)\right)\right]^{p_{k}}+\sum_{2}\left[f\left(q\left(\Delta^{m} \frac{x_{k}}{\pi_{k}}-L\right)\right)\right]^{p_{k}}\right) \\
\geq & \frac{1}{\lambda(n)} \sum_{1}\left[f\left(q\left(\Delta^{m} \frac{x_{k}}{\pi_{k}}-L\right)\right)\right]^{p_{k}} \\
\geq & \frac{1}{\lambda(n)} \sum_{1}[f(\varepsilon)]^{p_{k}} \\
\geq & \frac{1}{\lambda(n)} \sum_{1} \min \left([f(\varepsilon)]^{\inf p_{k}},[f(\varepsilon)]^{G}\right) \\
= & \frac{1}{\lambda(n)}\left|\left\{k \leq \lambda(n): q\left(\Delta^{m} \frac{x_{k}}{\pi_{k}}-L\right) \geq \varepsilon\right\}\right| \min \left([f(\varepsilon)]^{\inf p_{k}},[f(\varepsilon)]^{G}\right) .
\end{aligned}
$$

Hence $x \in S\left(\Delta_{q}^{m}\right)$.

Theorem 3.3 Let $f$ be a bounded and $0<h=\inf p_{k} \leq p_{k} \leq \sup _{k} p_{k}=G<\infty$. Then $S\left(\Delta_{q}^{m}\right) \subset C_{c_{\pi}}^{\lambda}\left(\Delta^{m}, f, p, q\right)$.

Proof. Suppose that $f$ is bounded. Let $q \in Q, \varepsilon>0$ and let $\sum_{1}$ and $\sum_{2}$ was denoted in previous theorem. Since $f$ is bounded there exists an integer $K$ such that $f(x)<K$, for all $x \geq 0$. Then

$$
\begin{aligned}
& \frac{1}{\lambda(n)} \sum_{k=1}^{\lambda(n)}\left[f\left(q\left(\Delta^{m} \frac{x_{k}}{\pi_{k}}-L\right)\right)\right]^{p_{k}} \\
\leq & \frac{1}{\lambda(n)} \sum_{1}\left[f\left(q\left(\Delta^{m} \frac{x_{k}}{\pi_{k}}-L\right)\right)\right]^{p_{k}}+\frac{1}{\lambda(n)} \sum_{2}\left[f\left(q\left(\Delta^{m} \frac{x_{k}}{\pi_{k}}-L\right)\right)\right]^{p_{k}} \\
\leq & \frac{1}{\lambda(n)} \sum_{1} \max \left(K^{h}, K^{G}\right)+\frac{1}{\lambda(n)} \sum_{2}[f(\varepsilon)]^{p_{k}} \\
\leq & \max \left(K^{h}, K^{G}\right) \frac{1}{\lambda(n)}\left|\left\{k \leq \lambda(n): q\left(\Delta^{m} \frac{x_{k}}{\pi_{k}}-L\right) \geq \varepsilon\right\}\right| \\
& +\max \left(f(\varepsilon)^{h}, f(\varepsilon)^{G}\right) .
\end{aligned}
$$

Hence $x \in C_{c_{\pi}}^{\lambda}\left(\Delta^{m}, f, p, q\right)$.

Theorem 3.4. $S\left(\Delta_{q}^{m}\right)=C_{c_{\pi}}^{\lambda}\left(\Delta^{m}, f, q\right)$ if and only if $f$ is bounded.

Proof : Let $f$ be bounded. By Theorem 3.2 and Theorem 3.3 we have $S\left(\Delta_{q}^{m}\right)=C_{c_{\pi}}^{\lambda}\left(\Delta^{m}, f, q\right)$.

Conversely, suppose that $S\left(\Delta_{q}^{m}\right)=C_{c_{\pi}}^{\lambda}\left(\Delta^{m}, f, q\right)$ and $f$ is unbounded. Then there exists a positive sequence $\left(t_{\lambda(n)}\right)$ with $f\left(t_{\lambda(n)}\right)=(\lambda(n))^{2}, n=1,2,3, \ldots$. If we choose

$$
\Delta^{m} \frac{x_{k}}{\pi_{k}}=\left\{\begin{array}{cc}
t_{\lambda(n)}, & k=(\lambda(n))^{2}, n=1,2,3, \ldots \\
0, & \text { otherwise }
\end{array}\right.
$$


then we have

$$
\frac{1}{\lambda(n)}\left|\left\{k \leq \lambda(n): q\left(\Delta^{m} \frac{x_{k}}{\pi_{k}}-L\right) \geq \varepsilon\right\}\right| \leq \frac{\sqrt{\lambda(n)}}{\lambda(n)} \longrightarrow 0 \text { as } n \longrightarrow \infty .
$$

Hence $x_{k} \longrightarrow 0\left(S\left(\Delta_{q}^{m}\right)\right)$, but $x \notin C_{c_{\pi}}^{\lambda}\left(\Delta^{m}, f, q\right)$ for $q=|x|$ and $X=\mathbb{C}$. Indeed let $q=|x|$ and $X=\mathbb{C}$. Then

$$
\begin{aligned}
& \Delta^{m} \frac{x_{k}}{\pi_{k}}=\left(\Delta^{m} \frac{x_{1}}{\pi_{1}}, \Delta^{m} \frac{x_{2}}{\pi_{2}}, \Delta^{m} \frac{x_{3}}{\pi_{3}}, \Delta^{m} \frac{x_{4}}{\pi_{4}}, \ldots, \Delta^{m} \frac{x_{9}}{\pi_{9}}, \Delta^{m} \frac{x_{10}}{\pi_{10}}, \ldots, \Delta^{m} \frac{x_{16}}{\pi_{16}}, \ldots\right) \\
= & \left(t_{\lambda(1)}, 0,0, t_{\lambda(2)}, 0, \ldots, 0, t_{\lambda(3)}, 0, \ldots, 0, t_{\lambda(4)}, 0, \ldots, 0, t_{\lambda(5)}, 0, \ldots\right) .
\end{aligned}
$$

Let $s_{n}=\frac{1}{\lambda(n)} \sum_{k=1}^{n} f\left(\left|\Delta^{m} \frac{x_{k}}{\pi_{k}}\right|\right)$. Then

$$
\begin{aligned}
s_{(\lambda(n))^{2}} & =\frac{1}{(\lambda(n))^{2}}\left((\lambda(1))^{2}+(\lambda(2))^{2}+(\lambda(3))^{2}+\ldots+(\lambda(n))^{2}\right) \\
& =\frac{\lambda(n)(\lambda(n)+1)(2 \lambda(n)+1)}{6(\lambda(n))^{2}}
\end{aligned}
$$

by (7). Now the subsequence $\left(s_{(\lambda(n))^{2}}\right)$ of $\left(s_{\lambda(n)}\right)$ is unbounded. Therefore $\left(s_{\lambda(n)}\right) \notin C_{\left(\ell_{\infty}\right)_{\pi}}\left(\Delta^{m}, f, q\right)$ and hence $\left(s_{\lambda(n)}\right) \notin C_{c_{\pi}}^{\lambda}\left(\Delta^{m}, f, q\right)$. Then contradicts to $S\left(\Delta_{q}^{m}\right)=C_{c_{\pi}}^{\lambda}\left(\Delta^{m}, f, q\right)$.

Consequently, the results obtained in [1] are generalized by using $C_{\lambda}$-method and modulus function with more general and weaker conditions. The analogous results in [31] may be obtained by this method.

\section{Acknowledgements}

The authors wish to thank the referee for his carefully reading of the manuscript and valuable suggestions.

\section{REFERENCES}

[1] Altin, Y. and Et, M. Generalized difference sequence spaces defined by a modulus function in a locally convex space, Soochow J. Math., 31(2) (2005), 233-243.

[2] Armitage D.H. and Maddox I.J. A new type of Cesáro mean, Analysis 9 (1989), 195-204.

[3] Bektaş, Ç. A. ; Et, M. and Çolak, R. Generalized difference sequence spaces and their dual spaces, J. Math. Anal. Appl. 292(2) (2004), 423-432.

[4] Et, M. ; Altinok, H. and Altin, Y. On some generalized sequence spaces. Appl. Math. Comput. 154(1) (2004), 167-173

[5] Et, M. Strongly almost summable difference sequences of order $m$ defined by a modulus, Studia Sci. Math. Hungar. 40(4) (2003), $463-476$.

[6] Et, M. and Çolak, R. On some generalized difference sequence spaces, Soochow J. Math. 21(4) (1995), 377-386.

[7] Et, M. Generalized Cesàro difference sequence spaces of non-absolute type involving lacunary sequences, Appl. Math. Comput. 219(17) (2013), 9372-9376.

[8] Fast, H. Sur la convergence statistique, Colloq. Math., 2 (1951), 241-244.

[9] Fridy, J. A. On the statistical convergence, Analysis, 5 (1985), 301-313.

[10] Güngör, M. and Et, M. $\Delta^{r}$-strongly almost summable sequences defined by Orlicz functions, Indian J. Pure Appl. Math. 34(8) (2003), 1141-1151.

[11] Johann B. Classical and Modern methods in summability, Oxford University Press, Oxford, 2006.

[12] Jürimäe, E. Matrix mappings between rate-spaces and spaces with speed, Acta Comm. Univ.Tartu. 970(1994), 29-52.

[13] Jürimäe, E. Properties of domains of mappings between rate-spaces and spaces with speed, Acta Comm. Univ.Tartu. 970(1994), 53-64.

[14] Kızmaz, H. On certain sequence spaces, Canad Math. Bull., 24(2) (1981), 169-176.

[15] Maddox I. J. Sequence spaces defined by a modulus, Math. Proc. Camb. Philos. Soc. 100 (1986), 161-166.

[16] Maddox I. J. Elements of Functional Analysis, Chambridge Univ. Press, 1970 (first edition).

[17] Malkowsky, E. and Parashar, S. D. Matrix transformations in spaces of bounded and convergent difference sequence of order m, Analysis 17 (1997), 87-97. 
[18] Mursaleen, M. ; Çolak, R. and Et, M. Some geometric inequalities in a new Banach sequence space, J. Inequal. Appl. 2007, Art. ID 86757, $6 \mathrm{pp}$.

[19] Nakano H. Concave modulars, J. Math. Soc. Japan 5 (1953), 29-49.

[20] Nuray, F. and Savaş, E. Some new sequence spaces defined by a modulus function, Indian J. Pure Appl. Math. 24(11) (1993), 657-663.

[21] Osikiewicz J. A. Equivalance Results for Cesáro Submethods, Analysis 20 (2000), 35-43.

[22] Ruckle W. H. FK spaces in which the sequence of coordinate vectors is bounded, Canad. J. Math. 25 (1973), 973-978.

[23] Šalát, T. On statistically convergent sequences of real numbers, Math. Slovaca 30 (1980), 139-150.

[24] Schoenberg, I. J. The integrability of certain functions and related summability methods, Amer. Math. Monthly 66 (1959), $361-375$.

[25] Sikk, J. Matrix mappings for rate-space and \$ \scr K\$-multipliers in the theory of summability. Tartu Riikl. Ül. Toimetised 846 (1989), 118-129.

[26] Tripathy B.C. and Chandra P. On Some Generalized Difference Paranormed Sequence Spaces Associated with Multiplier Sequences Defined by Modulus Function, Anal. Theory Appl. 27(1) (2011), 21-27

[27] Wilansky A., Summability Through Functional Analysis, North Holland, 1984.

[28] Wilansky A., Functional Analysis, Blaisdell Press, 1964.

[29] Wilansky A., Modern Methods in Topolgical Vector Spaces, McGraw-Hill,1978.

[30] Zeller K., Allgemeine Eigenschaften von Limitierungsverfahren, Math. Z. 53 (1951), 463-487.

[31] Vairamanickam, K Rao Chandrasekhara ,K and Sivaraman1, B., Generalized difference rate sequence spaces defined by Orlicz Functions, International Journal of Advance Research, IJOAR.org, vol.2, Issue 2, February 2014. 